\title{
LA PERSONALIDAD DEL «HORIZONTE» NECROPOLIS DEL CERRO DE LA ENCANTADA
}

Helena Romero Salas

Proyecto "ARXEOS"

Aunque en la actualidad es sobradamente, y bien, conocida la significación que el Yacimiento arqueológico del Cerro de la Encantada, en Granátula de Calatrava (Ciudad Real), ofrece para el conocimiento del Bronce de La Mancha, gracias a la especial personalidad de las diversas estructuras que forman parte y caracterizan a sus distintos conjuntos de restos (viviendas, construcciones defensivas, construcciones funerarias, necrópolis, etc...), queremos hacer aquí un resumen y ofrecer las conclusiones del que sea muy posiblemente su "horizonte" más representativo y el que marca en gran parte esa especial personalidad a la que hacemos referencia, así como ese carácter propio y prácticamente único que tiene este Yacimiento entre los muchos conocidos del entorno geográfico-cultural de La Mancha: el «horizonte" necrópolis, al que, hasta la fecha y desde 1977, año en que se realiza la primera Campaña de Excavaciones en el Cerro de la Encantada, pertêthecen un total de treinta y siete sepulturas, que representan hoy por hoy la mayor concentración conocida de estructuras de este tipo, científicamente excavadas, en los Yacimientos de la Región Manchega.

Estas sepulturas se encuentran situadas indistintamente tanto en el Sector A como en el Sector B del Yacimiento, perteneciendo 24 de ellas al primero, y las 13 restantes al segundo.

En cuanto a los tipos, por su relación arquitectónica con otras estructuras, existen dos grupos claramente diferenciados, por una parte el de las sepulturas exentas, $y$, por otra, el de las adosadas a un edificio o lo que hemos llamado "Complejon. En cada uno de estos grupos se han distinguido a su vez, otras subdivisiones, o subtipos, en función de las diferencias particulares que presentan, y ello en relación con su técnica constructiva, su situación topográfica, la morfología que las caracteriza, etc..., resultando de todo ello la siguiente clasificación:

EXENTAS: sepulturas de lajas, sepulturas de mampostería, sepulturas de pithoi, sepulturas de fosa.

ADOSADAS: sepultura de mampostería y sepultura de pithos.

Pertenecen al segundo de los grupos la sepultura número 1, un pithos depositado en el interior de una estructura de mampostería de planta rectangular, y que 
aparece adosado al Edificio Funerario del "Complejo 1", y la sepultura número 7/1, construida en mampostería, de planta igualmente rectangular y adosada al Edificio Funerario conocido como "Complejo 7" (1).

Dentro del primer grupo, el de las exentas, las sepulturas de lajas representan el conjunto más numeroso, pues del indicado total de las 37 tumbas existentes, 16 pertenecen a este tipo; se trata de tumbas cuyas "paredes" están construidas con piedras de gran tamaño, generalmente de forma rectangular, bien labradas, sensiblemente aplanadas, y colocadas verticalmente en el suelo. Construidas con una sola hilada, algunas aprovechan la existencia o afloramientos de la roca natural para constituir alguna de sus "paredes".

Le siguen a este grupo, numéricamente hablando, las sepulturas de mampostería, representadas por 11 tumbas, que se caracterizan por tener sus paredes construidas con piedras rectangulares de mediano tamaño, generalmente bien trabajadas y alisadas en su cara interna, están colocadas en posición horizontal. Estas sepulturas conservan de dos a cinco hiladas; en algunos casos, como ocurre con las sepulturas de lajas, han aprovechado para su construcción la presencia de la roca natural recortada, bien usada como parte de una pared, completando el resto con piedras, o bien para formar la "construcción" de una de sus paredes por entero.

Atendiendo a la "calidad" de su técnica constructiva, hemos dividido a las sepulturas de mampostería en dos tipos, las que están construidas cuidadosamente, es decir, aquéllas en cuya construcción se han utilizado piedras regularmente rectangulares, alisadas, muy bien trabadas entre sí y bien alineadas en sus muros, y aquellas otras que se han construido con piedras de forma irregular, más grandes de tamaño que las anteriores, mal trabadas entre sí, y sin guardar apenas un buen alineamiento, al primero de los tipos, al "cuidado", pertenecen las sepulturas número $6,7 / 1,11 / 1,17,23,36$ y 38 , las de técnica "descuidada" son las número 2 , $7 / 2,9,11 / 2$ y 15 .

Las sepulturas de pithoi son aquéllas en las que se ha utilizado para el enterramiento una vasija de cerámica, de mayor o menor tamaño, que luego se ha depositado, siempre en posición horizontal, bien sobre el suelo directamente, rodeada y calzada con piedras, o en el interior de una fosa, o de una covacha, o de otra estructura arquitectónica. Existen en la necrópolis del Cerro de la Encantada 7 sepulturas de pithoi.

Por último, las sepulturas menos representadas son las de fosa, tipo en el que se ha incluido la única tumba conocida en este Yacimiento hasta la fecha, y que se caracterizan porque no tienen ningún tipo de protección, ni utilizan para su delimitación estructura alguna.

(1) Nieto Gallo, G.; Sánchez Meseguer, J. et Alii: El Cerro de la Encantada. Granátula de Calatrava (Ciudad Real); E.A.E. (113), 1980, Madrid.

Nieto Gallo, G.; Sánchez Meseguer, J. et Alii: El Cerro de la Encantada. Granátula de Calatrava. Campaña 1979; N.A.H. (17) págs. 7 y ss., 1983, Madrid.

Sanz del Cerro, E. y Sánchez Meseguer, J.: Sepulturas de «mampostería en la Provincia de Ciudad Real: una aproximación a su estudio y paralelismos; I Congreso de Historia de Castilla-La Mancha, 1985, Ciudad Real. En prensa. 
En lo relacionado con el rito, todas las sepulturas excavadas hasta el momento en el Cerro de la Encantada son de inhumación. Se han diferenciado en lo que concierne al rito, el enterramiento individual, simple, que siempre corresponde a un solo individuo inhumado y colocado en posición fetal, y al que hemos llamado enterramiento 1, y el enterramiento doble caracterizado por la presencia de dos individuos, uno, del que suele aparecer todo su esqueleto y prácticamente siempre en posición fetal, y otro como a modo de "paquete», con sus restos físicos nunca anatómicamente completos y amontonados y descolocados, que por lo general aparece depositado a la altura de la pelvis, o de las rodillas, del individuo primero, y que hemos llamado enterramiento 2 .

En el caso de las sepulturas en pithoi, algunas de ellas presentan el tipo de enterramiento en inhumación individual simple, apareciendo todos los restos del cadáver en posición fetal, mientras que en otros solamente aparecen tan sólo parte de los restos, y estos descolocados, lo que nos hace pensar en que pudiera tratarse de inhumaciones secundarias, es decir que después de una primera inhumación o de un descarnamiento del individuo, se colocó en el pithos solamente una parte de él.

En lo que respecta a la orientación que presentan estos enterramientos, hay una clara intencionalidad de situarlos en dirección E-W, colocando la cabeza hacia el primer punto y, la mayor parte de los inhumados, con la cara vuelta hacia el Sur.

En cuanto a los ajuares que ofrecen estas sepulturas, y en lo que a su naturaleza material se refiere, son, por orden de abundancia: cerámica, metal (solamente aparecen objetos fabricados en cobre y sus aleaciones y en plata, muy probablemente obtenida en las proximidades del propio Yacimiento), hueso, piedras y alimento.

En relación con el número de objetos, los vasos cerámicos que ạparecen en los enterramientos como ajuar funerario nunca superan la cantidad de dos; entre las armas y útiles de metal, el número máximo de piezas nunca es superior a tres. Las cuentas de collar son las más numerosas cuando forman parte del ajuar, llegando a encontrarse hasta cinco o seis en algunas sepulturas.

Las formas cerámicas correspondientes a los 32 vasos existentes en el ajuar de las sepulturas, se reparten entre vasos carenados, ollas, cuencos y vasos troncocónicos.

Para los objetos de metal, como se ha dicho líneas arriba, hemos empleado los términos de "útiles" y de "armas", lo que se explica porque dentro de los útiles se incluyen, convencionalriente hablando, los punzones, y entre las armas están incluidos los puñales y las puntas de flecha.

Los útiles de hueso no han aparecido, hasta el momento, formando parte del ajuar en ninguno de los tipos de sepulturas reseñados. En cuanto a los útiles de piedra, los objetos que, como ajuar o parte integrante del mismo, han proporcionado las sepulturas, son los siguientes: alisador, denticulado, y un hacha, o azuela, de las llamadas "votivas".

Entre los objetos de adorno, los hay que están fabricados en piedra, en hueso y también en metal, y pueden ser cuentas de collar, colgantes, elementos de collar, zarcillos y brazaletes.

En la tabla sinóptica número 1 se recogen todos estos datos, así como la relación de los materiales que forman el ajuar de las distintas sepulturas, con el fin 
de ofrecer una visión general con la que se pueden seguir las notas o características más representativas que a continuación exponemos, y que son el resumen y resultado del análisis y estudio realizado por nosotros en otro lugar (2), sobre los enterramientos del Cerro de La Encantada, siempre alentados en su elaboración por el Profesor Nieto Gallo, al que queremos rendir con estas líneas un póstumo y afectuoso homenaje como discípulos suyos.

No deberá llamar la atención la ausencia, en la tabla sinóptica que presentamos, de las sepulturas número 33,34 y 35 , porque ello se debe al hecho de que, en un primer momento, y tras su aparición durante los trabajos de excavación, fueron clasificadas y numeradas como tales tumbas, ya que se trataba de unas estructuras formalmente similares a las restantes sepulturas, y que, una vez excavadas en su totalidad, pudimos comprobar que no correspondían a lugares de enterramiento, ni contenían restos humanos, siendo por lo tanto 37 el total de tumbas, aunque debemos recordar que al presentar dos de ellas, la número 7 y la número 11 , dos enterramientos superpuestos, se las inventaría como $7 / 1$ y $7 / 2$ y $11 / 1$ y 11/2, respectivamente.

Analizadas todas las características que ofrecen las sepulturas de la necrópolis del Cerro de la Encantada, podemos señalar de ellas, las siguientes notas:

\section{Sepulturas de lajas}

En las sepulturas de lajas las formas cerámicas predominantes son los vasos carenados, éstos se pueden encontrar indistintamente en sepulturas de este tipo, y tanto en el Sector A como en el Sector B del Yacimiento, cuando aparecen dos vasos la asociación característica es vaso carenado-vaso carenado, solamente en un caso encontramos la asociación vaso carenado-cuenco. El tipo más frecuente, casi general, que ofrecen estos vasos es el de presentar el diámetro del hiperboloide igual al diámetro del cuenco y la altura del hiperboloide mayor que la del cuenco.

En relación con los objetos de metal (útiles o armas) que como parte de su ajuar contienen estas sepulturas, son los punzones los únicos representados (y nunca en número superior a uno en la tumba en que existen), si bien hay que señalar que aunque los vasos carenados existan en los dos Sectores del Yacimiento, como ya hemos dicho, cuando la asociación es de un punzón con un vaso cerámico, en este caso, sólo aparecen en las sepulturas del Sector A del Yacimiento.

Entre los objetos de adorno, en metal, de los dos brazaletes argentíferos existentes como vinculables a las ofrendas funerarias, uno de ellos aparece formando parte del ajuar en una sepultura de este tipo y asociado a un vaso cerámico; esta sepultura fue descubierta en el Sector B del Yacimiento.

(2) En nuestra Memoria de Licenciatura titulada: El «Horizonte Necrópolis» del Cerro de La Encantada y los “Complejos funerarios", en curso de publicación dentro de la Serie "ORETUM" del Museo de Ciudad Real. 
CERRO DE LA ENCANTADA: SEPULLUURAS, AJUARES

\begin{tabular}{|c|c|c|c|c|c|c|c|c|c|c|c|c|c|}
\hline \multicolumn{14}{|c|}{ CERRO DE LA ENCANTADA: SEPULLTURAS, AJUARES } \\
\hline TIPO & NUH. & CERAM. 1 & CERAR. 2 & METAL 1 & METAL. 2 & METAL 3 & AD.PIEDRA & AD. HUESO & AD. METAL & Q.PIEDRA & RITO & EDAD & SEC \\
\hline Fosi & 28 & V. CARENADOC & V. CARENADO & PUKzON CU & PUNzON CU & FRG. PUNZDN СU & & & & . & SIMPLE & SENIOR & B \\
\hline INDT. & 37 & OLLITA & & $\cdot$ & & & & & & & SIMPLE & SENIOR & B \\
\hline LAJAS & $\begin{array}{l}03 \\
04 \\
10 \\
12 \\
13 \\
14 \\
18 \\
20 \\
21 \\
24 \\
25 \\
27 \\
29 \\
30 \\
31 \\
32 \\
\end{array}$ & $\begin{array}{l}\text { V. CAREMADO } \\
\text { V. CAREMADO } \\
\text { V. CARENADO } \\
\text { V. CARENADO } \\
\text { OLLA } \\
\text { V. CARENADO } \\
\text { V. CAREWADD } \\
\text { V. CARENADO }\end{array}$ & $\begin{array}{l}\text { V. CARENADO } \\
\text { V. CARENADO } \\
\text { V. TRONCOCONICO } \\
\text { CUENCO }\end{array}$ & $\begin{array}{l}\text { Punzow cU } \\
\text { Punzon cU } \\
\text { punzon Cu }\end{array}$ & . & & CUENTA & & Brazalete AG & & $\begin{array}{l}\text { SIMPLE } \\
\text { DOBLE } \\
\text { DOBLE } \\
\text { VIOLADA } \\
\text { SIMPLE } \\
\text { SIMPLE } \\
\text { SIFPLE } \\
\text { DOBLE } \\
\text { VIOLAPA } \\
\text { SILPLE } \\
\text { SIMPLE } \\
\text { SIMPLE } \\
\text { SIMPLE } \\
\text { VIOLADA } \\
\text { SIMPLE } \\
\text { SIMPLE }\end{array}$ & $\begin{array}{l}\text { SEMIOR } \\
\text { SEMIOR } \\
\text { SENIOR } \\
v \\
\text { SENIOR } \\
\text { SENIOR } \\
\text { SENIOR } \\
\text { SENIOR } \\
v \\
v \\
\text { SENIOR } \\
\text { INF ANS } \\
\text { SENIOR } \\
\text { SEMIOR } \\
v \\
\text { SENIOR } \\
\text { INFANS }\end{array}$ & $\begin{array}{l}A \\
B \\
B \\
A \\
A \\
A \\
A \\
A \\
A \\
A \\
B \\
B \\
A \\
B \\
A \\
A\end{array}$ \\
\hline MANPOSTERIA & $\begin{array}{l}06 \\
0711 \\
07 / 2 \\
09 \\
11 / 1 \\
11 / 2 \\
15 \\
17 \\
22 \\
23 \\
36 \\
38\end{array}$ & $\begin{array}{l}\text { CUENCO } \\
\text { OLLA } \\
\text { CUENCO } \\
\text { OLLA } \\
\text { CUENCO } \\
\text { V. CARENADO } \\
\text { V. CARENADO }\end{array}$ & CUENCO & $\begin{array}{l}\text { PUNAL } \\
\text { PUNAL CU } \\
\text { PUNALL CU } \\
\text { PUNZON CU } \\
\text { PUKALL CU } \\
\text { PUNTA FLECHA }\end{array}$ & $\begin{array}{l}\text { PUnzoN CU } \\
\text { PUnzoN CU }\end{array}$ & & $\begin{array}{l}\text { EL.COLLAR CUENTAS } \\
\text { CUENTAS }\end{array}$ & COLGAHTE & & $\begin{array}{l}\text { DENIICULADO HACHA } \\
\text { AL ISADDR }\end{array}$ & $\begin{array}{l}\text { DOBLE } \\
\text { SIMPLE } \\
\text { DOBLEE } \\
\text { SIMPLE } \\
\text { SIFPLE } \\
\text { SIMPLE } \\
? \\
\text { DOBLLE } \\
\text { SIMPLE } \\
\text { SIMPLE } \\
\text { SIMPLE } \\
\text { VIOLADA }\end{array}$ & $\begin{array}{l}\text { SENIOR } \\
? \\
\text { SENIOR } \\
\text { SENIOR } \\
\text { SENIOR } \\
\text { SENIOR } \\
? \\
\text { SENIOR } \\
\text { SENIOR } \\
\text { SENIOR } \\
\text { SENIOR } \\
v\end{array}$ & $\begin{array}{l}\text { A } \\
A \\
\hat{A} \\
\hat{A} \\
\mathbf{B} \\
\hat{A} \\
\hat{A} \\
\hat{A} \\
A \\
A\end{array}$ \\
\hline PITHOS & $\begin{array}{l}01 \\
02 \\
05 \\
08 \\
16 \\
19 \\
26\end{array}$ & $\begin{array}{l}\text { V. TRONCOCONICO } \\
\text { OLLA } \\
\text { v. CARENADO } \\
\text { v. ChREMADO }\end{array}$ & $\begin{array}{l}\text { OLLITA } \\
\text { FRG. OLLA }\end{array}$ & & & & . & & $\begin{array}{l}\text { BRAZALETE AG } \\
\text { C. TUBULAR CU } \\
\text { 2ARCILIO CU } \\
\text { FRG.CU }\end{array}$ & & $\begin{array}{l}\text { SIMPLE } \\
\text { SIMPLE } \\
\text { SIMPLE } \\
\text { SIMPLE } \\
\text { SIMPLE } \\
\text { SIMPLE } \\
\text { SIFPLE }\end{array}$ & $\begin{array}{l}\text { INFANS } \\
\text { SENIOR } \\
\text { SENIOR } \\
\text { INF ANS } \\
\text { INF ANS } \\
\text { INFANS } \\
\text { INF ANS }\end{array}$ & $\begin{array}{l}\text { A } \\
\text { B } \\
\text { B } \\
\text { A } \\
\text { B } \\
\text { B } \\
\text { A }\end{array}$ \\
\hline
\end{tabular}


En cuanto a los objetos de adorno fabricados en piedra, las cuentas y los elementos de collar, siempre van asociados a los punzones, y sólo aparecen en el Sector $\mathrm{A}$, a su vez estos objetos, adorno-punzones, van asociados siempre a ollas.

Este tipo de sepulturas carecen de útiles de piedra entre los objetos de su ajuar.

Las sepulturas de lajas que contienen enterramientos dobles se caracterizan por tener siempre vasos carenados, pudiendo presentar más de uno de estos vasos cerámicos; el tipo de rito doble puede estar representado en sepulturas situadas en el Sector A y en el Sector B, nunca se asocian con metal, tampoco aparecen asociados con objetos de adorno, y, por último, los restos óseos de estos enterramientos pertenecen a individuos adultos.

En cuanto a los enterramientos simples, al igual que las sepulturas que contienen enterramientos del tipo de rito doble, se asocian con vasos carenados (la mayoría), y están situadas, excepto una que aparece en el Sector B, en el Sector A; en metal, y a diferencia de las de rito doble, se asocian a punzones y a objetos de adorno de piedra y metal; los individuos inhumados en el interior de estas sepulturas son adultos, excepto un enterramiento, cuyos restos pertenecen a un individuo tipo «infans".

\section{Sepulturas de mampostería}

En este tipo de sepulturas, de las que solamente aparecen con ajuares las localizadas en el Sector A, encontramos como objetos cerámicos más característicos los cuencos, que sí se asocian a otros objetos, lo hacen con otro cuenco, siendo a su vez morfológicamente distintos los que aparecen en las sepulturas de mampostería cuidada, de los contenidos en los de mampostería descuidada, perteneciendo los clasificados de casquete a las primeras y los hiper-ovoides a las segundas.

Los vasos carenados de estas sepulturas, a diferencia de los aparecidos en las de lajas, se caracterizan por tener el diámetro y la altura del hiperboloide mayor que la del cuenco.

En relación con el metal, los puñales son los objetos más característicos entre los ajuares de este tipo de enterramientos en el Cerro, y van asociados en su mayor parte a los cuencos, y en un sólo caso a una olla, también pueden ir asociados a otros objetos metálicos, en este caso a punzones.

Entre los objetos de adorno, estas sepulturas carecen de objetos fabricados en metal, mientras que en hueso sólo es una la sepultura que contiene un colgante, y presenta la siguiente asociación: vaso carenado-punta de flecha. Por estas características hay que señalar que esta sepultura se sale un poco de los patrones que la mayoría de ellas presentan. En piedra, las cuentas y elementos de collar siempre van asociadas a punzones, a su vez estos objetos, adornos-punzones van asociados siempre a ollas.

Son muy pocas las sepulturas que entre sus ajuares contienen útiles de piedra; cuando aparecen, son característicos de las de mampostería, y lo hacen asociados el denticulado y el hacha pulimentada a una olla, puñal, punzón, cuentas y ele- 
mento de collar, mientras que el alisador se asocia a cuenco y puñal. De cualquier modo, como podemos observar, aparecen asociados a los ajuares en los que el puñal metálico forma parte de la ofrenda.

En cuanto al tipo de rito empleado en estas sepulturas, utilizan tanto el de inhumación doble, como el simple; en relación con el primero aparecen en sepulturas asociadas a cuencos, y pueden contener más de un vaso cerámico. Cuando presentan metal, se asocian a puñales y en un sólo caso, además les acompaña un punzón, no se asocian con objetos de adorno, pero sí, aunque en un número muy escaso a útiles de piedra; las sepulturas que contienen enterramientos de tipo simple se encuentran asociadas a vasos carenados, pero siempre contienen un vaso cerámico; en metal se asocian a puñales, punzones y en un caso a una punta de flecha, también pueden ir asociados a objetos de adorno de piedra y hueso, igualmente pueden contener o asociarse con útiles de piedra, aunque éstos están representados en un número escaso de sepulturas. Los individuos inhumados en las tumbas de mampostería son todos adultos.

\section{Sepulturas de phitoi}

En las sepulturas de pithoi predominan más las ollas y pueden aparecer tanto en el Sector A como en el B del Yacimiento. Se asocian, o bien con otra olla o con un vaso troncocónico.

Estas sepulturas se caracterizan por carecer de útiles y armas de metal, tampoco aparecen entre sus ajuares útiles de piedra, ni adornos de hueso y piedra.

Acompañando a los vasos cerámicos sólo encontramos adornos de metal.

En cuanto al rito se refiere, presentan el tipo individual o simple; de entre los siete enterramientos existentes, cinco pertenecen a "infans", y sólo dos a individuos adultos. Han sido encontrados indistintamente en el Sector A y en el B del Yacimiento, pero hay que señalar que en el Sector A se trata de enterramientos de niños, mientras que en el Sector B, aparecen tanto niños, como individuos adultos.

Los pithoi están colocados siempre en posición horizontal, como es frecuente en los enterramientos de la llamada zona argárica, aunque allí también hay algunos de niños que aparecen en posición vertical; en el Cerro de la Encantada sólamente el situado en la grieta de una roca, la sepultura núm. 26 , se encontró en esa posición.

En relación con la situación del cadáver dentro del pithos, usan en la Encantada las dos formas, es decir, bien con la cabeza en el fondo, como se utiliza en el Egeo, o bien colocada en la boca del pithos como en Anatolia (3), todo ello independientemente de que se encuentren en el Sector A o en el B, y de que se trate de un individuo "infans" o "senior".

(3) Schubart, H.: Relaciones Mediterráneas de la Cultura del Argar, Zaphyrus XXVI, págs. 331 y ss., 1976. Salamanca. 
Presentan como nota característica la existencia de una piedra, más fina de grosor que las empleadas en las estructuras que lo rodean, que hace las veces de tapadera.

\section{Sepulturas de fosa}

La única sepultura de fosa, existente hasta la fecha en el Cerro de La Encantada, se caracteriza por contener el enterramiento, en inhumación simple, de un individuo adulto, al que se asocia un ajuar formado por dos vasos carenados, del mismo tipo que los que aparecen en las sepulturas de mampostería, si bien conviene destacar el que las carenas de los que ahora comentamos, son más bajas que las de los aparecidos entre los de las restantes sepulturas de la Necrópolis.

No contiene en su ajuar, ni armas ni objetos de adorno, así como tampoco útiles de piedra.

En cuanto al metal se refiere, este tipo de sepultura se caracteriza por la presencia en ella, formando parte del mismo, de tres punzones, siendo éste el único caso que conocemos en el Cerro de La Encantada, en que aparezcan tres objetos como parte integrante del ajuar de una sepultura.

Como resumen de todo lo expuesto hasta el momento, consideramos que el conjunto de enterramientos, tumbas, ajuares, etc..., que constituyen el «Horizonte $\mathrm{Ne}$ crópolis" del Cerro de la Encantada, pese a la diversidad morfológica que ofrecen y que pueden analizarse en la figura número 2 , representan a un homogéneo contexto cultural, el Bronce de La Mancha, enriquecido precisamente por esa misma diversidad, más atribuible a diferencias de carácter social y momentos temporales (4) que a falta de personalidad histórica del resto del poblado, o los objetos metálicos, o los de adorno que aparecen entre las cosas del mundo de los vivos, y las del mundo de los muertos, mundo que por otra parte presenta evidentes analogías de grupos de objetos y de tumbas en la relación presencia-ausencia y ajuares-tipos de sepulturas, lo que nos hace afianzarnos más en la idea ya expuesta, de la existencia de diferencias sociológicas y cronológicas dentro de ese "Horizonte", diferencias a las que también pueden, sin ninguna duda, haberse adicionado influencia y "aires" culturales de otras áreas peninsulares contemporáneas (5), pero nunca en calidad y cantidad tal que hayan podido enmascarar la personalidad cultural del Bronce de La Mancha.

(4) Romero Salas, H. y Sánchez Meseguer, J.: Facies Necrópolis del Cerro de La Encantada: aproximación a una estratigrafía. I Congreso de Historia de Castilla-La Mancha. 1985, Ciudad Real. En prensa.

(5) Romero Salas, H.; Sánchez Meseguer, J. y Sanz del Cerro, E.: La Encantada: Bronce de La Mancha o Bronce Argárico...? I Congreso de Historia de Castilla-La Mancha. 1985, Ciudad Real. En prensa. 


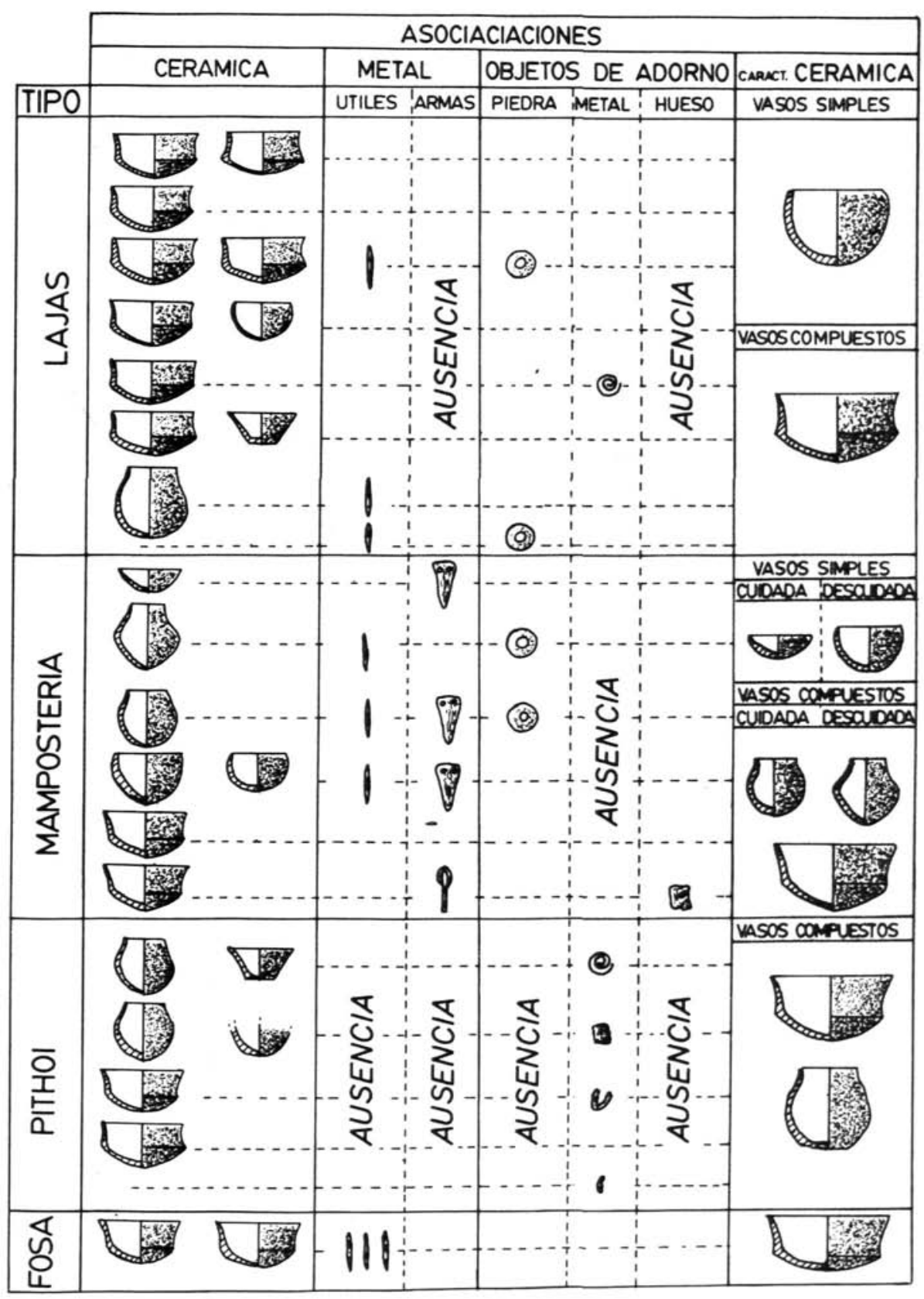

